\title{
Familial autosomal recessive bestrophinopathy: identification of a novel variant in BEST1 gene and the specific metabolomic profile
}

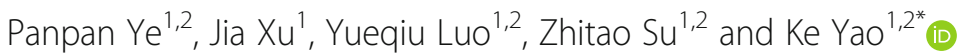

\begin{abstract}
Background: Autosomal recessive bestrophinopathy (ARB) is a retinal degenerative disorder caused by BEST1 mutations with autosomal recessive inheritance. We aim to map a comprehensive genomic and metabolomic profile of a consanguineous Chinese family with ARB.

Methods: Ophthalmic examinations were performed on the affected patients with ARB. The proband was screened for potential causative mutations in a panel with 256 known retinal disease genes by using target capture sequencing. The related mutation was further validated and segregated in the family members by Sanger sequencing. In silico prediction tools were used for pathogenicity assessment. A UHPLC-MS/MS metabolomic analysis was performed to explore the disease-associated metabolic feature.

Results: The affected patients from this family were characterized by low vision, the presence of subretinal fluid, macular edema, and hyperopia with coincidental angle closure. DNA sequencing identified a novel missense mutation in the BEST1 gene c.646G > A (p.Val216lle) of the proband. Sanger sequencing further confirmed the mutation. The missense mutation was co-segregation across the pedigree and predicted to be deleterious by SIFT (0.017). The blood metabolic profiles were highly similar among all family members probably because of the same lifestyle, habitat and genomic background. However, ARB patients presented a significant deregulation of metabolites, such as citric acid, L-Threonic acid, and eicosapentaenoic acid.
\end{abstract}

Conclusions: We identified a novel disease-associated variant in the BEST1 gene as well as a disease-specific metabolic feature in familial ARB. Our findings helped improve the understanding of ARB mechanisms.

Keywords: Autosomal recessive bestrophinopathy, BEST1, Mutation, Metabolomics

\section{Background}

Mutations of the BEST1 gene can cause a series of retinal degenerative diseases which are named as the "bestrophinopathies" [1]. The most common of these diseases clinically is Best vitelliform macular dystrophy (BVMD; OMIM 153700), also known as Best disease [1]. It is characterized by the deposition of bilateral yellowish yolk-like lesions in the macula [1]. BVMD is mostly inherited in an autosomal dominant type by mutations

\footnotetext{
* Correspondence: xlren@zju.edu.cn

${ }^{1}$ Eye Center, Second Affiliated Hospital, School of Medicine, Zhejiang University, Hangzhou, China

${ }^{2}$ Eye Hospital, Zhejiang University, Hangzhou, China
}

of the BEST1 gene [1]. An allelic disease, autosomal recessive bestrophinopathy (ARB; OMIM 611809), has been first reported by Burgess $\mathrm{R}$ [2]. It is also caused by BEST1 mutation with autosomal recessive inheritance [3-5]. The clinical phenotype can be different from that of BVMD. The features of ARB present with multifocal yellow subretinal deposits, subretinal fluid, macular edema, and hyperopia with coincidental angle closure. People with ARB demonstrate a decrease in vision during the first 10 years of their life.

The exact function of the BEST1 gene remains elusive, but it is speculated to be involved in coding for an anion channel, mainly a chloride and bicarbonate channel, or 
be a regulator of $\mathrm{Ca}^{2+}$ channels in the retinal pigment epithelium (RPE) [6]. So far, more than 250 mutations have been explored in bestrophinopathies [1]. In ARB, almost half of the mutations are located between residues 312 and 315 [2]. The most common mutation for ARB is p.R15H in several families of European ethnicity and p.R255W in Chinese ethnicity $[1,7,8]$. However, the whole genomic map of ARB and the underlying mechanism remain far from being completely understood.

The metabolome affects biological and physiological processes through modulation of genetic transcription, translation, and interactions with environmental exposures [9]. Therefore, the metabolites are closely linked with genetics and also related to the phenotype [10]. Because of the rapid development in biological techniques and bioinformatics, the metabolome has been used to not only identify biomarkers for diagnosis but also provide a therapeutic strategy in diseases $[11,12]$. In retinal health and disease, some groups have performed metabolomics for disease diagnosis and potential target intervention [10]. For instance, Li et al. identified plasma metabolites as biomarkers for the diagnosis and progression staging of diabetic retinopathy [13]. Laíns et al. also successfully revealed the metabolomics profile of different stages of age-related macular degeneration [14]. In the present study, we conducted a metabolomics study using blood samples in a consanguineous Chinese ARB family including three affected patients. We aimed to explore the disease-associated genomic and metabolic feature in order to better understand the etiology of ARB and provide a potential intervention strategy.

\section{Methods}

\section{Clinical diagnosis}

All research involved in this study adhered to the tenets of the Declaration of Helsinki and was approved by the Institutional Review Board of Second Affiliated Hospital of Zhejiang University (2019-133) (May, 2019). Informed written consent was obtained from all participating individuals in this study.

The patients were examined at Eye Center, Second Affiliated Hospital, School of Medicine of Zhejiang University, China. They underwent detailed ophthalmic evaluation, including best correct visual acuity (BCVA), slit-lamp biomicroscopy, dilated indirect ophthalmoscopy, anterior chamber (ultrasound biomicroscopy, UBM; SUOER UBM scan SW-3200), wide-field retinal imaging (Optos 200Tx, Marlborough, MA, USA), optical coherence tomography (OCT) (Heidelberg HRT II, Heidelberg, Germany), and fundus fluorescein angiography (FFA) (Heidelberg HRT II, Heidelberg, Germany) examinations.

\section{DNA library preparation and target sequencing}

Genomic DNA of the proband and the available family members was extracted from peripheral blood by using a DNA isolation kit (Qiagen, Hilden, Germany). A precapture library was prepared by using Kapa LTP library prep kit (Kapa Biosystems, Wilmington, USA) and then was captured on a custom capture panel (Agilent Sureselect, USA) which containing 256 known retinal disease genes (Additional file 1: Table S1). The enriched DNA library was sequenced on Illumina Xten Analyzers (San Diego, USA) for 150 cycles per read to generate paired-end reads. An average of $172.82 \mathrm{X}$ in target region was achieved, and $97.86 \%$ of the target region was covered by $10 x$.

\section{Bioinformatics analysis and sanger sequencing}

After the sequencing step, raw reads were aligned to the human genome reference (hg19) by using the BurrowsWheeler Aligner (Wellcome Trust Sanger Institute, Cambridge, UK). Single-nucleotide variants (SNVs) and Insertions and Deletions (InDels) were called by AtlasSNP2 and Atlas-Indel, respectively. SNVs and InDels were filtered against the ExAC, gnomAD, HGVD, CHARGE, 1000 Genome, and UK10K databases and the internal database of Clinbytes Inc. with an allele frequency cutoff of 0.5 and $0.1 \%$ for recessive and dominant variants, respectively. Variants were annotated using Annotate Variation (ANNOVAR). Conservation analysis of the related homologous proteins in the mutation site was performed using the UCSC Genome Browser database. In silico gene function prediction software (e.g., SIFT, PolyPhen2, and FATHMM) was used for pathogenicity assessment. The variants were further validated and segregated by Sanger sequencing from all available family members. PCR primer sets were designed via Primer3, and the products were sequenced on an ABI 3700XL Genetic Analyzer (Thermo Fisher, USA).

\section{UHPLC-MS/MS metabolomics analysis}

The patients' peripheral blood was collected from the available family members, and plasma was prepared for LC-MS/MS analysis, which was performed using the Vanqiush UHPLC system (Thermo Fisher) coupled with an Orbitrap Q Exactive HF-X mass spectrometer (Thermo Fisher) operating in the data-dependent acquisition mode by Novogene Co., Ltd. (Beijing, China). The detailed information has been provided previously [15]. The samples were divided into three groups according to the genotype in chr11:61725867 loci. The plasma metabolites were compared between the homozygous variation group (A/A, $n=2)$ and the wild-type group (G/G, $n=3)$, as well as between the homozygous variation group $(\mathrm{A} / \mathrm{A}, n=2)$ and the heterozygosity group (A/G, $n=2)$.

\section{Results}

It is a consanguineous Chinese family with Han Chinese ancestry. The proband is a 34-year-old woman with progressive vision loss for over 20 years. Her BCVA was 
0.15 in the right eye and 0.1 in the left eye. Color funduscopy (CF) was normal, overall (Fig. 1a). The fundus autofluorescence (FAF) image showed annular hyperfluorescence around the posterior pole (Fig. 1b). OCT results revealed cystoid macular edema with subretinal fluid (Fig. 1c). The proband has an affected younger sister aged 32, with 20 years of progressive vision loss. Her BCVA was 0.1 in both eyes. Her clinical findings in CF, FAF, and OCT were similar to those of the proband. In addition, she had a shallower anterior chamber depth, and UBM showed that half of the anterior chamber angles were closed (Fig. 1d). FFA revealed mild fluorescence leakage beneath the macula (Fig. 1e). The proband also has an affected elder sister aged 36, with over 20 years of progressive vision loss. Both of her eyes underwent vitrectomy for retinal detachment and her visual acuity in both eyes was counting fingers. According to these abnormalities, ARB was suspected, and further genetic testing was performed in this family.

We performed the target capture sequencing of known retinal disease genes on the proband to identify possible mutation. A homozygous variation in the BEST1 gene was detected in the 256 gene coding regions associated with retinal diseases in the peripheral blood of the subjects. The variant in the BEST1 gene (chr11:61725867G > A) was confirmed in the proband. According to the splicing form of the proband (uc010rlu.1), the mutation BEST1 c.646G > A (p.V216I) was located in exon 7. This is a missense mutation resulting in a replacement of Valine by Isoleucine at amino acid position 216 . The $\mathrm{G}$ to $\mathrm{A}$ allele variant has not been reported in the ExAC database. It was predicted to be deleterious using SIFT (score: 0.017) and FATHMM (score: - 5.32) but not PolyPhen2 (score: 0.007) in dbNSFP (version3.0). All

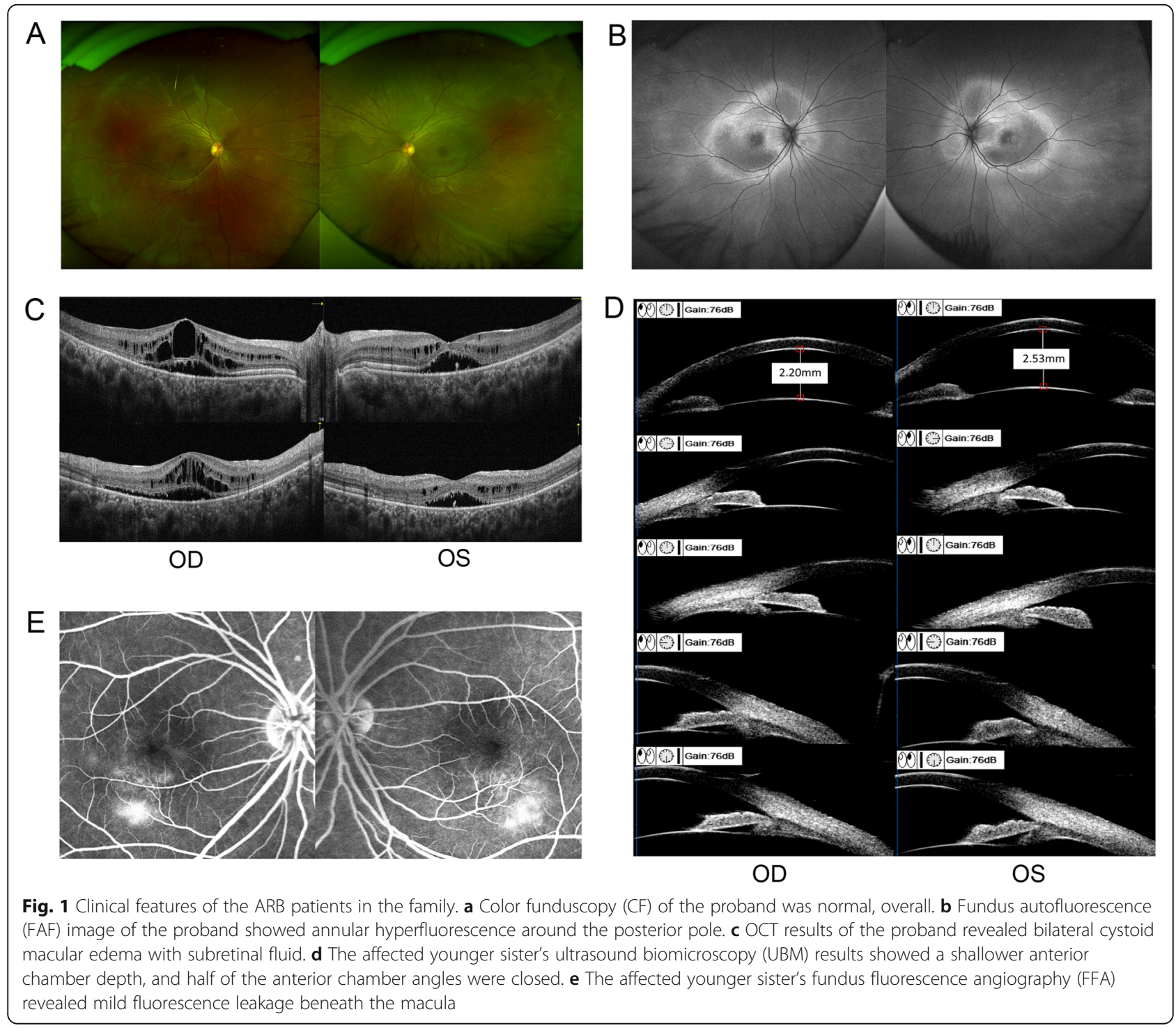


available family members were Sanger sequenced for the segregation analysis (Fig. 2). The results of the family separation and analysis showed that the proband, her fifth sister, and her younger sister were carrying the homozygous variation, and all of them had low vision. The mother and the son of the proband and the son of her younger sister carried the heterozygosity, and the clinical phenotype of the three was normal. The missense mutation was co-segregation across the pedigree and possessed strong amino acid conservation among different species (Fig. 3). Based on the clinical phenotype and genetic variation pattern, the patients were diagnosed with ARB.

We performed a metabolomic exploration to evaluate the potential inherited metabolic disorders, which could be diagnostic or therapeutic targets. The distribution of the metabolites is shown in Fig. 4a. We found a very similar blood metabolite profile in this family. Using a criterion $(P<0.05$, VIP $>1.0)$, there were $2.0-2.6 \%$ (fold change $>1.2$ ) and $1.2-2.0 \%$ (fold change $>2.0$ ) metabolites significantly differential expressed between the two groups (Table 1). Compared with her three wild-type sisters (G/G), the proband and her fifth sister, both of whom were of A/ A homozygous variant type, showed significantly down-regulated citric acid (fold change $>20$ ), flurothyl, L-Threonic acid, and eicosapentaenoic acid (EPA), etc., as well as up-regulated hydrogen sulfate, manidipine, 2,4-Dichlorobenzoyl-CoA, acetate, etc. (Fig. 4b). Compared with their parents (A/G), they (A/A) also presented significantly down-regulated citric acid and flurothyl, and up-regulated hydrogen sulfate and 2,4Dichlorobenzoyl-CoA. The enrichment analyses of differential expressed metabolites indicated the central role of citric acid in enriched metabolic pathways (Fig. 4c). Therefore, we recommended lemonade, which is rich in citric acid and vitamin $C$, for the ARB patients. After three months, OCT of the proband showed a decreased macular edema compared with the initial visit (Additional file 2: Figure S1), although no significant change was found in the FAF.

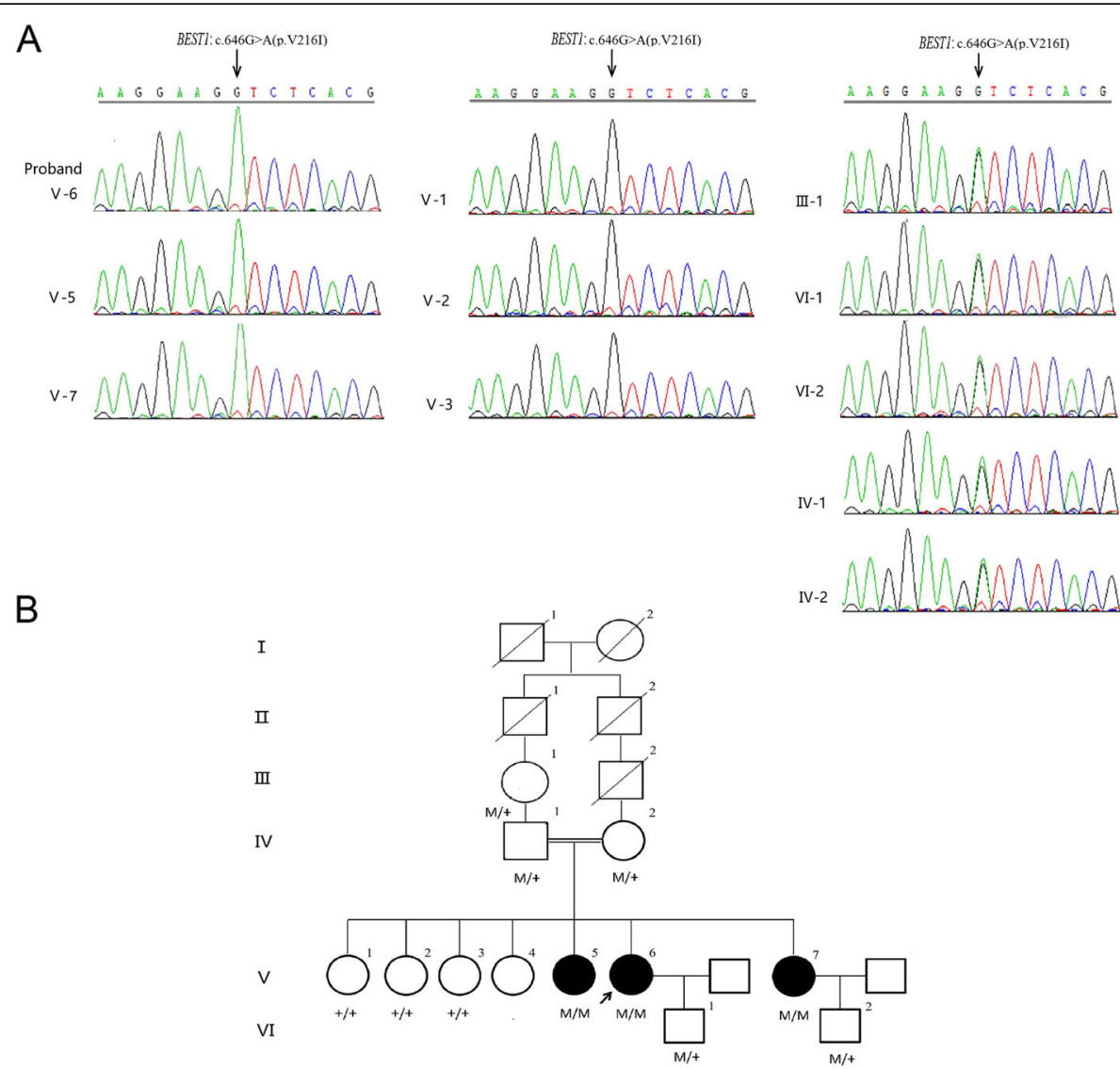

Fig. 2 A novel autosomal recessive mutation in the BEST1 gene c.646G > A (p.V216l) was identified in the family. a The results of family separation and analysis showed that the proband (V6), her fifth sister (V5), and her younger sister (V7) carry the homozygous variation. The grandmother (III-1), father (IV1), mother (IV2), and son (VI1) of the proband, her fourth sister (V4), and the son (VI2) of her younger sister carry the heterozygosity. The first sister (V1), the second sister (V2), and the third sister (V3) of the proband carry the wild-type gene. b Pedigree. Square symbols denote males, circle symbols denote females, solid symbols indicate the affected, open symbols indicate the unaffected, slash symbols indicate the deceased, an arrow below the symbol indicates the proband, = indicates consanguinity, and + indicates the wild type 

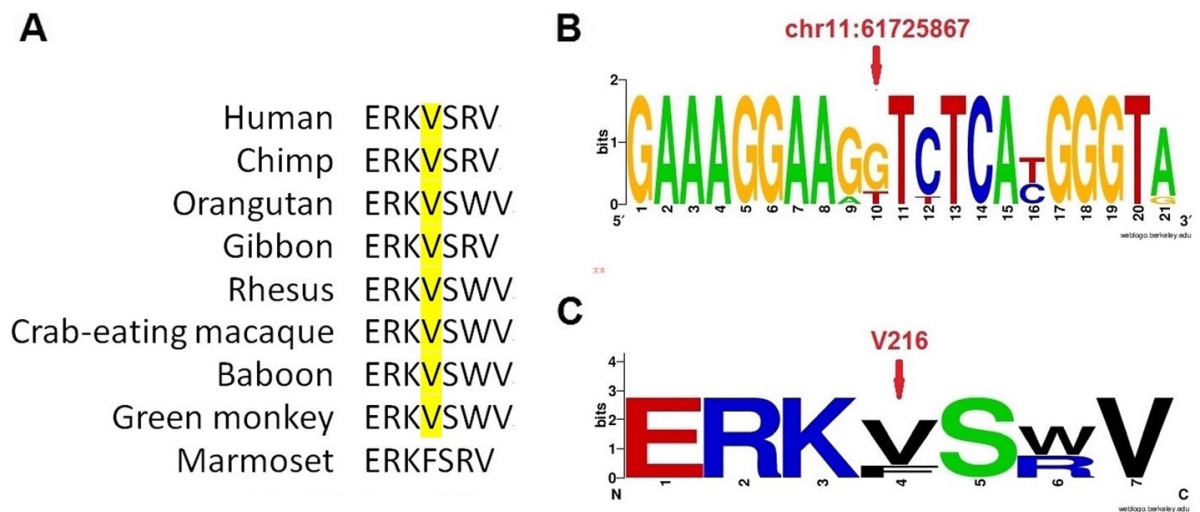

Fig. 3 Conservation analysis using the UCSC Genome Browser database. a Schematic representation of amino acid sequence alignment around the p.V216l variant in different species. b DNA WebLogo of the variant regions. c A conservational logo of amino acids of BEST1 protein position 216 (Valine) generated by WebLogo online tool

\section{Discussion}

We identified a novel homozygous variation in the BEST1 gene in a consanguineous family. This was a missense mutation and was co-segregation across the pedigree. In this family, three affected patients with the mutation c.646G > A (p.V216I) were homozygous and had a similar clinical phenotype. Three other members carrying the heterozygosity did not show any phenotype. This finding proved the fact that the inheritance pattern was autosomal recessive. Among betrophinopathies, ARB is presumed to be a retinal disease with a "null" phenotype for Best1 $[2,16]$. Based on the clinical abnormalities and gene variation pattern, the patients were diagnosed with ARB. However, EOG was unavailable at the time that patients were examined. That's a limitation of our workup.

The mutations of BEST1 can cause various clinical features [1]. To date, over 250 mutations have been explored through the BEST1 gene, and most mutations occur in the N-terminal part, which is highly conserved among various species [17]. However, the mutation spectrum of the Chinese population may be different from that of patients with other ethnicities. In a Chinese cohort with $20 \mathrm{ARB}$ patients, more than $1 / 3$ mutations $(9 / 22)$ in the BEST1 gene were located in exon 7-11, which encoded the C-terminal half of the protein [8]. The other mutations were mainly clustered in the first transmembrane domain and the intracellular regions [8]. In functional prediction analysis, the majority of diseasecausing variants were missense mutations [8], which was consistent with our results.

We also performed analyses to assess the association between the missense mutation and disease. In the pathogenicity prediction, SIFT and FATHMM but not PolyPhen2 predicted the mutation to be deleterious. SIFT and PolyPhen 2 are the most commonly used online tools for pathogenicity assessment, but they may provide different or even opposite results [18]. Therefore, we further analyzed the potential role of this mutation at the protein level. We found that the amino acid was highly conserved. This may indicate the importance of Valine at amino acid position 216 and support the pathogenicity of this mutation.

Although disease-associated nonsense and frameshift mutations predict a truncated and thus likely inactive protein, the molecular mechanism underlying the effect of missense mutation on protein function and the pathology of disease has not been well elucidated. Uggenti et al. showed that ARB-associated BEST1 protein was degraded via the ubiquitin-proteasome pathway using a stably transfected polarized epithelial cell model [19]. Milenkovic et al. further proved that the ARB-associated missense mutations triggered a strong and fast protein degradation process in the endoplasmic reticulum [20], thereby favoring a decreased stoichiometry of mutant versus normal BEST1 subunits in the assembly of the homo-pentameric BEST1 chloride channel.

In this study, we used a metabolome strategy to investigate a BEST1 mutation-caused disease. The family members presented very similar metabolic profiles probably because of the same lifestyle, environment, diet, habitat, and genomic background. However, we found a remarkable insufficiency of metabolites, such as citric acid, L-Threonic acid, and EPA, in the blood of the patients compared with that of their sisters. Citric acid or citrate is a key component of the TCA cycle; it serves as a pivotal regulator of intermediary energy metabolism and a chelator for divalent cations, such as like $\mathrm{Ca}^{2+}$, $\mathrm{Mg}^{2+}$, and $\mathrm{Zn}^{2+}$ [21]. It is uptaken by human RPE cells as a preferred nutrient [22], and its buffer is widely used in the in vivo retina experiments. L-Threonate acid is a metabolite of ascorbate (vitamin $\mathrm{C}$ ), which is highly concentrated in the retina, and plays an important role in physiological function, and protects RPE from oxidant 

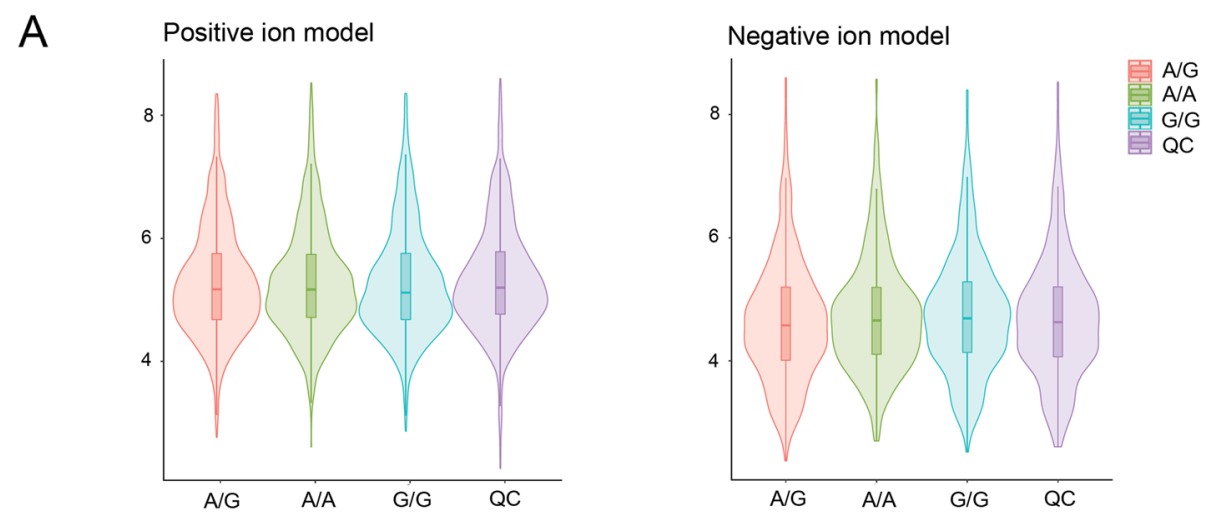

B Positive ion model A/A vs. G/G

Negative ion model A/A vs. G/G
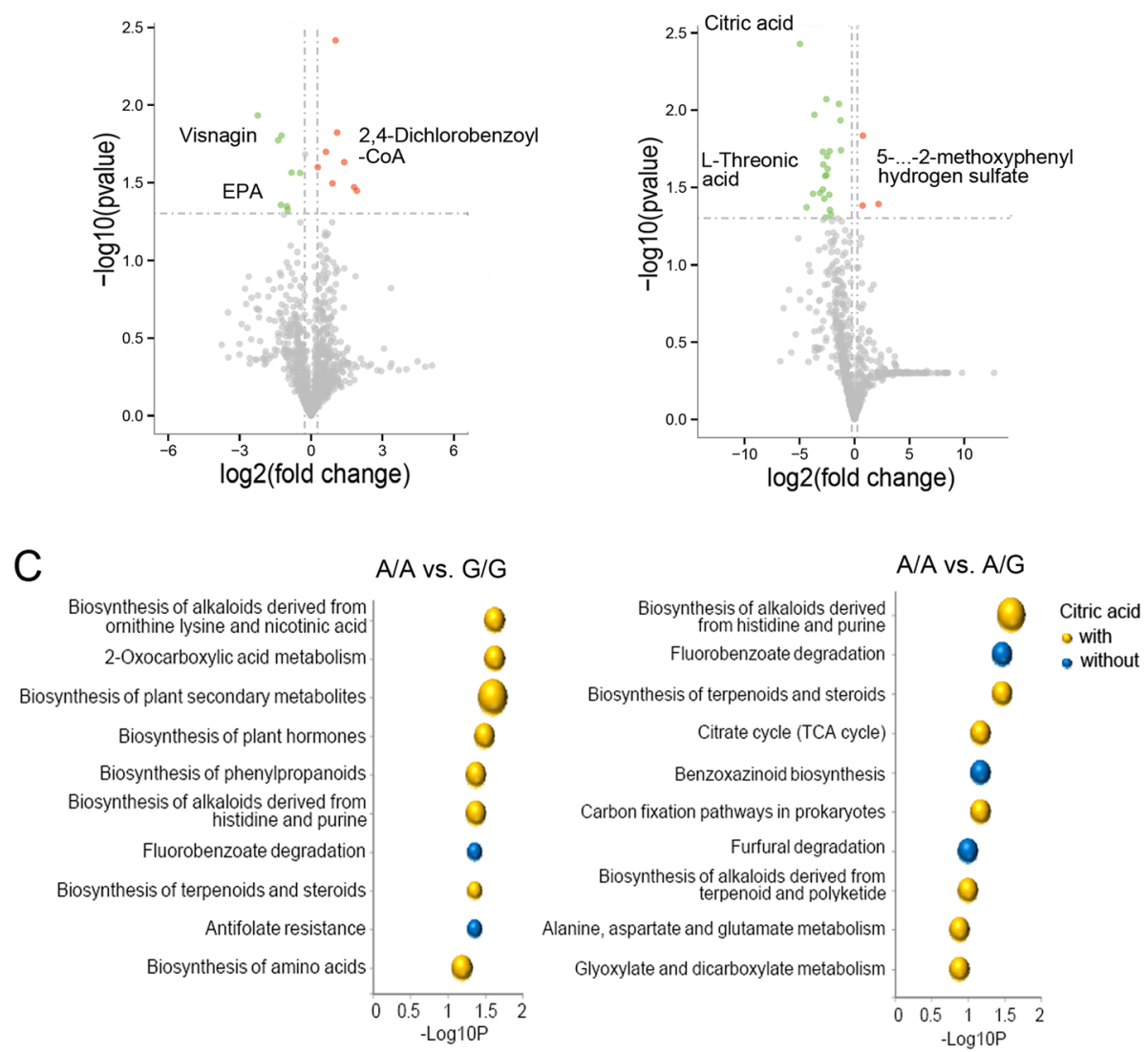

Fig. 4 Metabolic profile of ARB patients. a Distribution of metabolites in the whole family. $\mathbf{b}$ The significantly differential expressed metabolites (fold change $>1.2, P<0.05)$. $\mathbf{c}$ KEGG einrichment analysis of differential expressed metabolites between ARB patients $(A / A, n=2)$ and their wildtype sisters $(G / G, n=3)$ and between ARB patients $(A / A, n=2)$ and the heterozygosity group $(A / G, n=2)$. The size of the bubble represents the number of genes in each pathway

injury [23, 24]. A systemic review including 6150 participants demonstrated that this antioxidant vitamin could slow down the progression of age-related macular degeneration [25]. In addition, the biological importance of Omega-3 fatty acids (EPA and docosahexaenoic acid) in the development of the retina is well established [26]. A recent in vivo study showed that EPA supplementation could reduce lipofuscin granules and slow down the progression of retinal degeneration [27]. Therefore, a lack of these metabolites indicates defective nutrition of the human body, including the retina. According to the results of the metabolome, we recommended a dietary citric acid and vitamin $C$ supplementation in the ARB patients. We found an alleviation of macular edema after a short-term metabolic therapy. The results encouraged the patients to receive longer and more comprehensive 
Table 1 Numbers of significantly differential expressed metabolites (DEM) $(P<0.05, \mathrm{VIP}>1.0)$

\begin{tabular}{cllll}
\hline Comparison & $\begin{array}{l}\text { Total } \\
\text { metabolites } \\
(\mathrm{n})\end{array}$ & DEM (n) & $\begin{array}{l}\text { Up-regulated } \\
\text { DEM (n) }\end{array}$ & $\begin{array}{l}\text { Down- } \\
\text { regulated } \\
\text { DEM (n) }\end{array}$ \\
\hline Fold change > 1.2 & & & & \\
A_A.vs.G_G_pos & 990 & 16 & 8 & 8 \\
A_G.vs.G_G_pos & 990 & 24 & 17 & 7 \\
A_A.vs.A_G_pos & 990 & 18 & 14 & 4 \\
A_A.vs.G_G_neg & 670 & 25 & 3 & 22 \\
A_G.vs.G_G_neg & 670 & 19 & 6 & 13 \\
A_A.vs.A_G_neg & 670 & 14 & 4 & 10 \\
Fold change > 2.0 & & & & \\
A_A.vs.G_G_pos & 990 & 10 & 5 & 5 \\
A_G.vs.G_G_pos & 990 & 15 & 11 & 4 \\
A_A.vs.A_G_pos & 990 & 9 & 5 & 4 \\
A_A.vs.G_G_neg & 670 & 23 & 1 & 22 \\
A_G.vs.G_G_neg & 670 & 5 & 3 & 2 \\
A_A.vs.A_G_neg & 670 & 12 & 3 & 9 \\
\hline
\end{tabular}

nutrient supplementation (e.g., Omega-3 fatty acid) in order to meet their metabolic needs.

This study has limitations. First, the pathogenicity of the mutation was predicted by in silico tools. Further functional experiments are needed to prove the hypothesis. Second, the metabolites in the peripheral plasma may not reflect the changes occurring in the retina. Nevertheless, the present research is a pioneering study that explore the association between altered human metabolism and genetic disease. We believe that some genetic diseases could result in abnormal metabolism and nutritional deficiencies. However, whether nutrient supplementation could ameliorate or even correct the genetic diseases remains to be explored further.

\section{Conclusion}

We provided a new insight into the genomic profiling of the pathological changes in familial ARB. The exploration of the specific metabolism and nutrition status of the genetic disease sheds light on its underlying mechanism and pathophysiology and might provide a potential intervention strategy in the future.

\section{Supplementary information}

Supplementary information accompanies this paper at https://doi.org/10. 1186/s12881-020-0951-3.

Additional file 1: Table S1. The list of 256 known retinal disease genes. Additional file 2. Figure S1. The OCT results of the proband.

\section{Abbreviations}

ARB: Autosomal recessive bestrophinopathy; BCVA: Best correct visual acuity; BVMD: Best vitelliform macular dystrophy; CF: Color funduscopy;
EPA: Eicosapentaenoic acid; FAF: Fundus autofluorescence; FFA: Fundus fluorescein angiography; InDels: Insertions and Deletions; OCT: Optical coherence tomography; RPE: Retinal pigment epithelium; SNV: Singlenucleotide variants

\section{Acknowledgments}

None.

\section{Authors' contributions}

PY participated in study design, data acquisition, analysis and manuscript writing. JX participated in data acquisition and analysis. YL participated in data acquisition and analysis. ZS participated in data acquisition. KY participated in study conception, data interpretation and manuscript revision. All authors have read and approved the final manuscript.

\section{Funding}

This study was supported by Zhejiang Natural Science Foundation Project of China (No. LY18H120001) and National Natural Youth Science Foundation Project of China (No. 31500795).

Funding Role: The funding body has provided the funds for data collection and analysis and has no role in design of the study, data interpretation and manuscript writing.

\section{Availability of data and materials}

The datasets generated and/or analysed during the current study are available in the Google repository, https://drive.google.com/open?id=15KfYv4 VKKjw5MysQJxYJhNiK3dTTXHXI, or in the Baidu repository, https://pan.baidu. com/s/1jEXmZZtAMYTwufAEmmCZGw (accession number: ettq).

\section{Ethics approval and consent to participate}

This study was approved by the Institutional Review Board of Second Affiliated Hospital of Zhejiang University and in accordance with the 1964 Helsinki Declaration. Written informed consent was obtained for all participants or their guardians.

\section{Consent for publication}

Written informed consents were obtained from all individuals or their guardians.

\section{Competing interests}

The authors declare that they have no competing interests.

Received: 27 August 2019 Accepted: 7 January 2020

Published online: 22 January 2020

\section{References}

1. Johnson AA, Guziewicz KE, Lee CJ, Kalathur RC, Pulido JS, Marmorstein LY, Marmorstein AD. Bestrophin 1 and retinal disease. Prog Retin Eye Res. 2017; 58:45-69.

2. Burgess R, Millar ID, Leroy BP, Urquhart JE, Fearon IM, De Baere E, Brown PD, Robson AG, Wright GA, Kestelyn P, et al. Biallelic mutation of BEST1 causes a distinct retinopathy in humans. Am J Hum Genet. 2008;82(1):19-31.

3. Kinnick TR, Mullins RF, Dev S, Leys M, Mackey DA, Kay CN, Lam BL, Fishman $G A$, Traboulsi E, lezzi $R$, et al. Autosomal recessive vitelliform macular dystrophy in a large cohort of vitelliform macular dystrophy patients. Retina. 2011;31(3):581-95.

4. Iannaccone A, Kerr NC, Kinnick TR, Calzada Jl, Stone EM. Autosomal recessive best vitelliform macular dystrophy: report of a family and management of early-onset neovascular complications. Arch Ophthalmol. 2011;129(2):211-7.

5. Lee CS, Jun I, Choi SI, Lee JH, Lee MG, Lee SC, Kim EK. A novel BEST1 mutation in autosomal recessive Bestrophinopathy. Invest Ophthalmol Vis Sci. 2015:56(13):8141-50.

6. Guziewicz KE, Sinha D, Gomez NM, Zorych K, Dutrow EV, Dhingra A, Mullins RF, Stone EM, Gamm DM, Boesze-Battaglia K, et al. Bestrophinopathy: an RPE-photoreceptor interface disease. Prog Retin Eye Res. 2017;58:70-88.

7. Boon CJ, van den Born LI, Visser L, Keunen JE, Bergen AA, Booij JC, Riemslag FC, Florijn RJ, van Schooneveld MJ. Autosomal recessive bestrophinopathy: differential diagnosis and treatment options. Ophthalmol. 2013;120(4):809-20. 
8. Tian L, Sun T, Xu K, Zhang X, Peng X, Li Y. Screening of BEST1 gene in a Chinese cohort with BEST Vitelliform macular dystrophy or autosomal recessive Bestrophinopathy. Invest Ophthalmol Vis Sci. 2017;58(9):3366-75.

9. Levine B, Kroemer G. Biological functions of autophagy genes: a disease perspective. Cell. 2019;176(1-2):11-42.

10. Lains I, Gantner M, Murinello S, Lasky-Su JA, Miller JW, Friedlander M, Husain D. Metabolomics in the study of retinal health and disease. Prog Retin Eye Res. 2019;69:57-79.

11. Visweswaran M, Arfuso F, Warrier S, Dharmarajan A. Concise review: aberrant lipid metabolism as an emerging therapeutic strategy to target cancer stem cells. Stem Cells. 2019.

12. Van Treuren W, Dodd D. Microbial contribution to the human Metabolome: implications for health and disease. Annu Rev Pathol. 2019.

13. Li X, Luo X, Lu X, Duan J, Xu G. Metabolomics study of diabetic retinopathy using gas chromatography-mass spectrometry: a comparison of stages and subtypes diagnosed by Western and Chinese medicine. Mol BioSyst. 2011; 7(7):2228-37.

14. Lains I, Kelly RS, Miller JB, Silva R, Vavvas DG, Kim IK, Murta JN, Lasky-Su J, Miller JW, Husain D. Human plasma metabolomics study across all stages of age-related macular degeneration identifies potential lipid biomarkers. Ophthalmol. 2018;125(2):245-54.

15. Han C, Jiang YH, Li W, Liu Y, Qi ZQ. Study on the antihypertensive mechanism of Astragalus membranaceus and Salvia miltiorrhiza based on intestinal Flora-host metabolism. Evid Based Complement Alternat Med. 2019;2019:5418796.

16. Pomares E, Bures-Jelstrup A, Ruiz-Nogales S, Corcostegui B, Gonzalez-Duarte R, Navarro R. Nonsense-mediated decay as the molecular cause for autosomal recessive bestrophinopathy in two unrelated families. Invest Ophthalmol Vis Sci. 2012;53(1):532-7.

17. Boon CJ, Klevering BJ, Leroy BP, Hoyng CB, Keunen JE, den Hollander Al. The spectrum of ocular phenotypes caused by mutations in the BEST1 gene. Prog Retin Eye Res. 2009;28(3):187-205.

18. Cao Z, Zhu Y, Liu L, Wu S, Liu B, Zhuang J, Tong Y, Chen X, Xie Y, Nie K, et al. Novel mutations in HSF4 cause congenital cataracts in Chinese families. BMC Med Genet. 2018;19(1):150.

19. Uggenti C, Briant K, Streit AK, Thomson S, Koay YH, Baines RA, Swanton E, Manson FD. Restoration of mutant bestrophin-1 expression, localisation and function in a polarised epithelial cell model. Dis Model Mech. 2016;9(11): 1317-28.

20. Milenkovic A, Milenkovic VM, Wetzel CH, Weber BHF. BEST1 protein stability and degradation pathways differ between autosomal dominant BEST disease and autosomal recessive bestrophinopathy accounting for the distinct retinal phenotypes. Hum Mol Genet. 2018;27(9):1630-41.

21. Westergaard N, Waagepetersen HS, Belhage B, Schousboe A. Citrate, a ubiquitous key metabolite with regulatory function in the CNS. Neurochem Res. 2017:42(6):1583-8.

22. Chao JR, Knight K, Engel AL, Jankowski C, Wang Y, Manson MA, Gu H, Djukovic D, Raftery D, Hurley JB, et al. Human retinal pigment epithelial cells prefer proline as a nutrient and transport metabolic intermediates to the retinal side. J Biol Chem. 2017:292(31):12895-905.

23. Domith I, Socodato R, Portugal CC, Munis AF, Duarte-Silva AT, Paes-deCarvalho R. Vitamin $\mathrm{C}$ modulates glutamate transport and NMDA receptor function in the retina. J Neurochem. 2018;144(4):408-20.

24. Wei W, Li L, Zhang Y, Geriletu YJ, Xing Y. Vitamin C protected human retinal pigmented epithelium from oxidant injury depending on regulating SIRT1. ScientificWorldJournal. 2014;2014:750634.

25. Evans JR, Lawrenson JG. Antioxidant vitamin and mineral supplements for slowing the progression of age-related macular degeneration. Cochrane Database Syst Rev. 2012;11:CD000254.

26. SanGiovanni JP, Chew EY. The role of omega-3 long-chain polyunsaturated fatty acids in health and disease of the retina. Prog Retin Eye Res. 2005; 24(1):87-138.

27. Prokopiou E, Kolovos P, Kalogerou M, Neokleous A, Nicolaou O, Sokratous K, Kyriacou K, Georgiou T. Omega-3 fatty acids supplementation: therapeutic potential in a mouse model of Stargardt disease. Invest Ophthalmol Vis Sci. 2018:59(7):2757-67.

\section{Publisher's Note}

Springer Nature remains neutral with regard to jurisdictional claims in published maps and institutional affiliations.

\section{Ready to submit your research? Choose BMC and benefit from:}

- fast, convenient online submission

- thorough peer review by experienced researchers in your field

- rapid publication on acceptance

- support for research data, including large and complex data types

- gold Open Access which fosters wider collaboration and increased citations

- maximum visibility for your research: over $100 \mathrm{M}$ website views per year

At BMC, research is always in progress.

Learn more biomedcentral.com/submissions 